\title{
Changes in nutrition content and health claims post-implementation of regulation in Australia
}

\author{
Lyndal Wellard-Cole ${ }^{\top} \odot$, Rebecca Li ${ }^{2}$, Christine Tse ${ }^{2}$, Wendy L Watson ${ }^{1, *}$ and \\ Clare Hughes ${ }^{1}$ \\ 'Cancer Prevention and Advocacy Division, Cancer Council NSW, Woolloomooloo, NSW 2011 , Australia: \\ ${ }^{2}$ Discipline of Nutrition and Dietetics, School of Life and Environmental Science, Charles Perkins Centre, The University \\ of Sydney, Sydney, NSW 2006, Australia
}

Submitted 8 May 2019: Final revision received 22 October 2019: Accepted 21 November 2019: First published online 27 April 2020

\begin{abstract}
Objective: To determine whether there were changes in the prevalence or healthiness of products carrying claims post-implementation of Standard 1.2.7: Nutrition, Health and Related Claims in the Australia New Zealand Food Standards Code. Design: Observational survey of claims on food packages in three categories: nonalcoholic beverages, breakfast cereals and cereal bars. Nutrient profiling was applied to products to determine their eligibility to carry health claims under Standard 1.2.7. The Standard came into effect in 2013. The proportion of products carrying claims and the proportion of those not meeting the nutrient profiling criteria were calculated. A comparative analysis was conducted to determine changes between 2011 and 2016.

Setting: Three large metropolitan stores from the three major supermarket chains in Sydney, Australia were surveyed in 2011 and 2016.

Participants: All claims on all available products in 2016 ( $n$ 1737). Nutrition composition and ingredients were collected from the packaging.

Results: Overall in 2016, $76 \%$ of products carried claims and there were 7367 claims identified in the three food categories. Of products in 2016 with health claims, $34 \%$ did not meet nutrient profiling criteria. These may breach Standard 1.2.7. Comparison of 2011-2016 showed a significant increase in the number of products carrying claims (66 v. $76 \%, P<0.001)$.

Conclusions: The proportion of products carrying claims that do not meet nutrient profiling and consumers' tendency to infer health benefits from nutrition content claims warrants the regulation of all claims using the nutrient profiling. This will ensure consumers are not misled by claims on unhealthy food products.
\end{abstract}

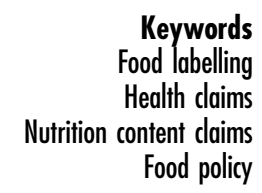

Keywords

Food labelling

claims

Food policy
Health and nutrition content claims are marketing tools used on food labels ${ }^{(1,2)}$. In Australia and New Zealand, the use of claims is regulated in Food Standard 1.2.7: Nutrition, Health and Related Claims of the Australia New Zealand Food Standards Code ${ }^{(3)}$.

Under Standard 1.2.7, nutrition content and health claims are regulated in a stepwise manner (Fig. 1) ${ }^{(3,4)}$. Nutrition content claims relate to the presence or absence of a nutrient or food component ${ }^{(3)}$, for example, 'low in fat'. Products carrying nutrition content claims must meet qualifying criteria for the nutrient being claimed, as set out in Standard 1.2.7 $7^{(3)}$. General-level health claims relate to a food-health relationship ${ }^{(3)}$, for example, 'low in fat for a healthy heart'. In addition to meeting the qualifying criteria, products carrying these claims must meet the minimum nutrition requirements set out in the Nutrient Profiling Scoring Criteria (NPSC), a score based on the energy, saturated fat, sugars, $\mathrm{Na}$, protein, fibre and fruit, vegetable, nut and legume content of the food ${ }^{(5)}$. The company making the claim must hold evidence that the food-health relationship exists ${ }^{(3)}$. High-level health claims are those on serious diseases or biomarkers of serious diseases that must be treated by a medical professional ${ }^{(3)}$, such as 'low in fat to reduce risk of heart disease'. In addition to the requirements for general-level health claims, high-level health claims must be pre-approved by Food Standards Australia New Zealand before the product goes to market $^{(3)}$. 


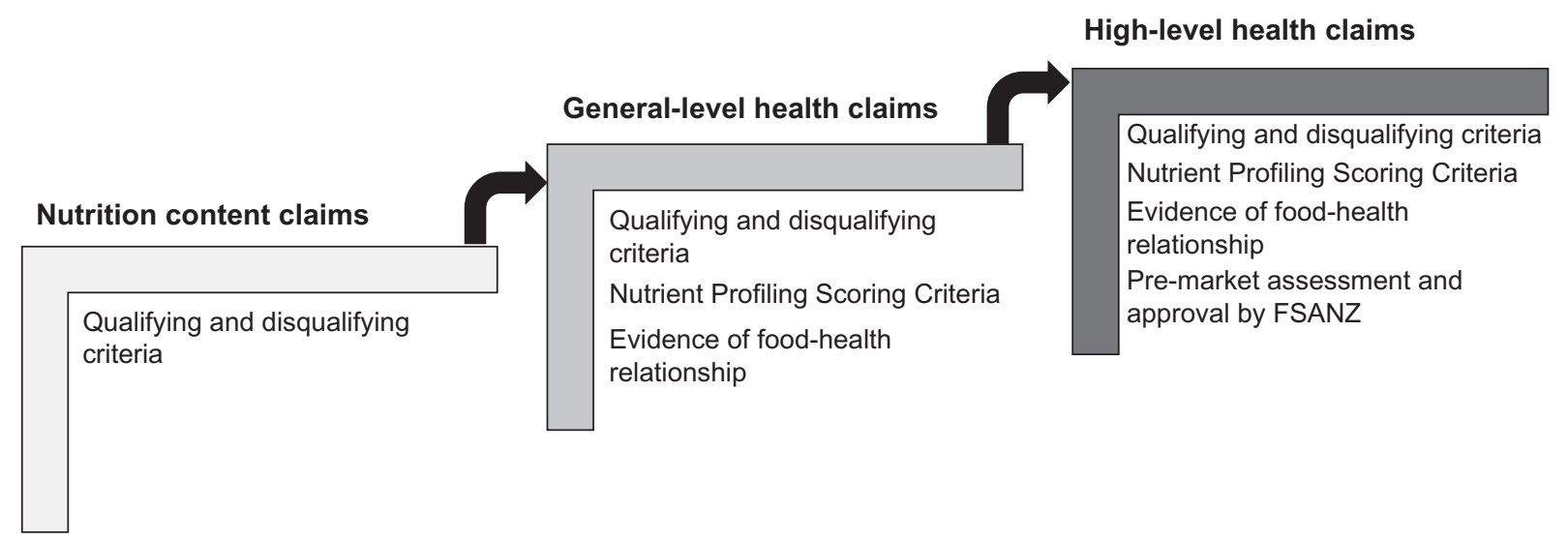

Fig. 1 Stepwise regulation of claims under the Australian New Zealand Food Standards Code ${ }^{(3,4)}$. FSANZ, Food Standards Australia New Zealand

In Australia, Standard 1.2.7 has been in effect since January 2013; however, food companies had a 3-year phase-in period and were required to comply by January $2016^{(6)}$. Prior to that, a voluntary code had been in place; however, instructions to develop regulation were raised with Food Standards Australia New Zealand in $2003^{(7)}$. Research conducted prior to the implementation of Standard 1.2.7 found that around one-third of products in specific food categories that carried health claims did not meet the NPSC and would not be allowed to do so once the Standard was introduced ${ }^{(7)}$. Other Australian research found that claims were prolific on ultra-processed foods, often containing high levels of added sugars ${ }^{(8)}$. Since the introduction of Standard 1.2.7, research in New Zealand found that $7 \%$ of products in eight food categories that did not meet the NPSC carried health claims and therefore may be non-compliant with Standard 1.2.7 $7^{(9)}$. However, this study only captured the front-of-pack of food products and therefore is likely to underestimate the prevalence of claims and compliance ${ }^{(9)}$.

There has not been any published research investigating whether the implementation of Standard 1.2.7 has seen a reduction in the proportion of products carrying claims that do not meet the NPSC. Therefore, this study builds on previous research ${ }^{(7)}$ to determine whether there were any changes in the prevalence or healthiness of products carrying claims post-implementation of Standard 1.2.7.

\section{Methods}

This study replicated the methods of the previous study ${ }^{(7)}$ conducted in 2011 prior to the implementation of Standard 1.2.7 to enable comparison of results. Ethics approval was not necessary as there were no human participants.

\section{Procedures}

Data were collected in March to May 2016 in NSW, in three large metropolitan outlets of the three largest supermarket chains in Australia. Together, these three chains account for nearly three quarters of the grocery market share in Australia ${ }^{(10)}$.

All available products in the non-alcoholic beverages (juices, soft drinks, energy drinks, cordials, sports drinks, plain and flavoured water), cereal bars (muesli, nut-based, yogurt/chocolate topped, fruit-filled) and breakfast cereal (ready-to-eat, oats and others requiring heating) categories were included ${ }^{(7)}$. These three categories were selected as they had been previously identified in Australian research as frequently carrying a range of health and/or nutrition content claims ${ }^{(1)}$. As some products were available in multiple supermarket chains, each product was only included once. Where multiple pack sizes were available, only the largest pack size of each product was included. Flavour variations of the same product (e.g., chocolate chip muesli bars and apricot muesli bars from the same brand) were considered as different products, and therefore each flavour variation was included.

Nutrition information, ingredients lists and claims were collected by photographing all facets of the product packages. Data were transcribed into Microsoft Excel spreadsheets. Data collected for each product were product name and variety, pack size, nutrition composition information per $100 \mathrm{~g} / \mathrm{ml}$ for energy, protein, saturated fat, sugars, $\mathrm{Na}$ and fibre (where available), ingredients lists and percentage fruit, vegetable, nut and legume content. All claims were recorded verbatim, including text, symbols and product names, but not trademarks. The pack was viewed from all sides, and each individual occurrence of a health or nutrition content claim was counted. Because of their extensive use on breakfast cereal and cereal bar packaging, wholegrains were included as nutrition content claims in the previous study and for consistency included in $2016^{(7)}$, despite not being covered by Standard 1.2.7(3).

The online Nutrient Profiling Scoring Calculator ${ }^{(5)}$ was used to determine whether products were eligible to carry health claims. For the purposes of this study, products that met the NPSC (and could therefore carry health claims) 
were referred to as 'healthy' and those that did not were deemed 'unhealthy'.

\section{Missing data}

In Australia, a product does not have to list dietary fibre unless it carries a fibre claim $^{(11)}$. Where this was missing off labels, manufacturers were contacted to provide the missing data. When information could not be obtained, estimations were made based on the average of comparable products in the sample ( $n 30,2 \%$ ).

Declaration of the percentage of fruit, vegetable, nut and legume content is only required on Australian food labels when these are characterising ingredients ${ }^{(12)}$. For products missing this information, estimations were made based on the ingredient order in the ingredients list, as they must be listed in descending order by weight ${ }^{(13)}$. The fruit, vegetable, nut and legume content only changes the final NPSC score when it is higher than $40 \%$; therefore, in cases where the percentage could not be estimated ( $n 35,2 \%$ ), the NPSC score was calculated using a value above and one below $40 \%$ fruit, vegetable, nut and legume content to determine whether eligibility to carry claims was affected. In the two products where eligibility was affected (0.01\%), fruit, vegetable, nut and legume content was estimated from a comparable product.

Claims were classified into nutrition content, generallevel health and high-level health claims. Claims were further categorised according to the nutrient or food component they related to. General nutrition, health and wellbeing claims (e.g. 'healthy', 'nutritious' or 'makes you feel better') were included as health claims as they implied health benefits.

\section{Reliability}

All data were entered and coded by two researchers, including calculating the NPSC scores. These were checked independently by a third researcher. When there was disagreement ( $n$ 19, 1\%), a fourth researcher reviewed the discrepancy and discussion was conducted until agreement was reached.

\section{Data analysis}

Descriptive analyses were conducted to describe the total number of claims and the most prevalent claims in each food category. Proportions of products in each category carrying claims were calculated. The proportions of products in each category that were classified as unhealthy by NPSC that carried claims were also determined.

Comparative analysis was conducted on the number and proportion of claims in 2011 (pre-implementation) compared with 2016 (post-implementation). Chi-squared tests were used to determine differences in the proportion of total and unhealthy products carrying claims. Statistical analysis was conducted using IBM SPSS version 19, and values of $P \leq 0.05$ were considered statistically significant.

\section{Results}

\section{Results from 2016 study}

Overall, there were 1737 products across the three food categories included in the sample. This included 996 beverages (57\%), 463 breakfast cereals (27\%) and 278 cereal bars (16\%). Of these, 1316 (76\%) carried at least one claim. The number and proportion of total products and products from each category can be seen in Table 1 .

In total, there were 7367 claims identified in the three food categories. Most claims ( $n$ 6073, $82 \%$ ) were nutrition content claims (Table 2 ). On average, there were 4.2 total claims per product (range $0-45$ per product), 3.5 nutrition content claims per product (range $0-45$ per product) and 0.7 health claims per product (range 0-14 per product).

A larger proportion of products carried nutrition content claims than health claims, particularly breakfast cereals ( $94 v$. $32 \%)$ and cereal bars (94v. 33\%). Breakfast cereals (32\%) and cereal bars (33\%) more commonly carried health claims than beverages (14\%). Of products carrying health claims, 131 (34\%) did not meet the NPSC. These products may be in breach of the Standard 1.2.7. All the products carrying high-level health claims met the NPSC. Of products carrying nutrition content claims, 338 (26\%) did not meet the NPSC, although that is not a requirement of Standard 1.2.7.

Nutrition content claims were grouped into twenty-one different nutrients and food components. The most common nutrition content claims were for vitamins and minerals ( $n$ 974, 16\% of all nutrition content claims), wholegrain claims ( $n$ 957, 16\%) and low sugar ( $n$ 921, $15 \%)$. By category, the most common nutrition content claims in beverages were low in sugar ( $n 756,38 \%)$, vitamins and minerals ( $n$ 493, $25 \%$ ) and energy ( $n$ 225, $11 \%$ ). However, the energy claims included both low energy (96\% of energy claims within the category) and high energy ( $4 \%)$. In cereals, the three most common claims were wholegrains ( $n$ 676, 23\%), high in fibre ( $n$ 593, $20 \%$ ) and vitamins and minerals ( $n$ 493, 17\%). In cereal bars, the three most common nutrition content claims were wholegrain ( $n$ 281, 24\%), high in fibre ( $n$ 238, $20 \%$ ) and gluten free ( $n$ 110, 9\%).

Health claims were grouped into thirty-four different food-health relationships. The most common health claims were for 'energy'/vitality' ( $n$ 382, 30 \%) 'hydration' ( $n$ 183, 14\%) and general health/well-being claims (e.g. 'protein for nourishment of the body' ( $n$ 182, $14 \%$ ). By category, the most common health claims in beverages were 'hydration' ( $n$ 183, 35\% of health claims), 'energy/vitality' ( $n$ 127, 25\%) and 'detox/cleanse/balance' ( $n$ 64, $12 \%$ ). In cereals, the most common were 'energy/vitality' ( $n$ 194, $34 \%$ ), general health/well-being ( $n 92,16 \%$ ) and 'digestive health' ( $n$ 60, $11 \%$ ). Similarly, in cereal bars, the two most common health claims were 'energy/vitality' and general health/well-being ( $n 61,30 \%$ and $n 34,17 \%$, respectively) and 'appetite suppression/satiety' and 'emotional wellbeing' (both $n$ 29, $14 \%$ ). 


\section{Public Health Nutrition}

Table 1 Number and \% within category of products carrying each type of claim and number and \% within category of products carrying claims that did not meet the Nutrient Profiling Scoring Criteria in 2011 and 2016

\begin{tabular}{|c|c|c|c|c|c|c|c|c|c|c|c|c|c|}
\hline \multirow[b]{2}{*}{ Claim type } & \multirow[b]{2}{*}{ Year } & \multicolumn{2}{|c|}{ Beverages } & \multirow[b]{2}{*}{$P$} & \multicolumn{2}{|c|}{$\begin{array}{c}\text { Breakfast } \\
\text { cereals }\end{array}$} & \multirow[b]{2}{*}{$P$} & \multicolumn{2}{|c|}{ Cereal bars } & \multirow[b]{2}{*}{$P$} & \multicolumn{2}{|c|}{ Total } & \multirow[b]{2}{*}{$P$} \\
\hline & & $n$ & $\%$ & & $n$ & $\%$ & & $n$ & $\%$ & & $n$ & $\%$ & \\
\hline \multicolumn{14}{|l|}{ Products carrying claims } \\
\hline All claims* & $\begin{array}{l}2011 \\
2016\end{array}$ & $\begin{array}{l}331 \\
617\end{array}$ & $\begin{array}{l}55 \\
62\end{array}$ & $0.01 \dagger$ & $\begin{array}{l}220 \\
437\end{array}$ & $\begin{array}{l}83 \\
94\end{array}$ & $<0.001 \dagger$ & $\begin{array}{l}128 \\
262\end{array}$ & $\begin{array}{l}77 \\
94\end{array}$ & $<0.001 \dagger$ & $\begin{array}{r}679 \\
1316\end{array}$ & $\begin{array}{l}66 \\
76\end{array}$ & $<0.001 \dagger$ \\
\hline Nutrition content claims & $\begin{array}{l}2011 \\
2016\end{array}$ & $\begin{array}{l}320 \\
585\end{array}$ & $\begin{array}{l}54 \\
59\end{array}$ & $0.04 \dagger$ & $\begin{array}{l}218 \\
436\end{array}$ & $\begin{array}{l}83 \\
94\end{array}$ & $<0.001 \dagger$ & $\begin{array}{l}128 \\
260\end{array}$ & $\begin{array}{l}77 \\
94\end{array}$ & $<0.001 \dagger$ & $\begin{array}{r}666 \\
1281\end{array}$ & $\begin{array}{l}65 \\
74\end{array}$ & $<0.001 \dagger$ \\
\hline All health claims & $\begin{array}{l}2011 \\
2016\end{array}$ & $\begin{array}{r}60 \\
141\end{array}$ & $\begin{array}{l}10 \\
14\end{array}$ & $0.02 \dagger$ & $\begin{array}{l}100 \\
150\end{array}$ & $\begin{array}{l}38 \\
32\end{array}$ & $0 \cdot 14$ & $\begin{array}{l}23 \\
91\end{array}$ & $\begin{array}{l}14 \\
33\end{array}$ & $<0.001 \dagger$ & $\begin{array}{l}183 \\
382\end{array}$ & $\begin{array}{l}18 \\
22\end{array}$ & $0.01 \dagger$ \\
\hline High level health claims $\ddagger$ & $\begin{array}{l}2011 \\
2016\end{array}$ & $\begin{array}{l}1 \\
1\end{array}$ & $\begin{array}{l}0.2 \\
0.1\end{array}$ & 1 & $\begin{array}{l}18 \\
19\end{array}$ & $\begin{array}{l}7 \\
4\end{array}$ & $0 \cdot 12$ & $\begin{array}{l}1 \\
2\end{array}$ & $\begin{array}{l}0.6 \\
0.7\end{array}$ & 1 & $\begin{array}{l}20 \\
22\end{array}$ & $\begin{array}{l}2 \\
1\end{array}$ & 0.2 \\
\hline \multicolumn{14}{|c|}{ Products carrying claims not meeting Nutrient Profiling Scoring Criteria } \\
\hline All claims* & $\begin{array}{l}2011 \\
2016\end{array}$ & $\begin{array}{r}65 \\
136\end{array}$ & $\begin{array}{l}20 \\
22\end{array}$ & 0.41 & $\begin{array}{l}37 \\
59\end{array}$ & $\begin{array}{l}17 \\
14\end{array}$ & 0.29 & $\begin{array}{l}101 \\
172\end{array}$ & $\begin{array}{l}79 \\
66\end{array}$ & $0.01 \dagger$ & $\begin{array}{l}203 \\
367\end{array}$ & $\begin{array}{l}30 \\
28\end{array}$ & 0.35 \\
\hline Nutrition content claims & $\begin{array}{l}2011 \\
2016\end{array}$ & $\begin{array}{r}54 \\
108\end{array}$ & $\begin{array}{l}17 \\
19\end{array}$ & 0.59 & $\begin{array}{l}37 \\
59\end{array}$ & $\begin{array}{l}17 \\
14\end{array}$ & 0.24 & $\begin{array}{l}101 \\
171\end{array}$ & $\begin{array}{l}79 \\
66\end{array}$ & $0.01 \dagger$ & $\begin{array}{l}192 \\
338\end{array}$ & $\begin{array}{l}29 \\
26\end{array}$ & $0 \cdot 26$ \\
\hline All health claims & $\begin{array}{l}2011 \\
2016\end{array}$ & $\begin{array}{l}21 \\
55\end{array}$ & $\begin{array}{l}35 \\
39\end{array}$ & 0.64 & $\begin{array}{l}18 \\
15\end{array}$ & $\begin{array}{l}18 \\
10\end{array}$ & 0.09 & $\begin{array}{l}18 \\
61\end{array}$ & $\begin{array}{l}78 \\
67\end{array}$ & 0.45 & $\begin{array}{r}57 \\
131\end{array}$ & $\begin{array}{l}31 \\
34\end{array}$ & 0.51 \\
\hline High-level health claims $\ddagger$ & $\begin{array}{l}2011 \\
2016\end{array}$ & $\begin{array}{l}0 \\
0\end{array}$ & $\begin{array}{l}0 \\
0\end{array}$ & - & $\begin{array}{l}2 \\
0\end{array}$ & $\begin{array}{r}11 \\
0\end{array}$ & 0.23 & $\begin{array}{l}1 \\
2\end{array}$ & $\begin{array}{l}100 \\
100\end{array}$ & - & $\begin{array}{l}3 \\
2\end{array}$ & $\begin{array}{r}15 \\
9\end{array}$ & 0.66 \\
\hline
\end{tabular}

*Many products carried both nutrition content and health claims, so this is not a sum of these.

†Significant results, $P \leq 0.05 \chi^{2}$ tests.

$\ddagger$ This is a subset of all health claims. 
Table 2 Number and proportion of each type of claim

\begin{tabular}{|c|c|c|c|c|c|c|c|c|}
\hline \multirow[b]{3}{*}{ Food category } & \multicolumn{8}{|c|}{$n$ and $\%$ of total ${ }^{*}$} \\
\hline & \multicolumn{2}{|c|}{ Beverages } & \multicolumn{2}{|c|}{ Breakfast cereals } & \multicolumn{2}{|c|}{ Cereal bars } & \multicolumn{2}{|c|}{ Total } \\
\hline & $n$ & $\%$ & $n$ & $\%$ & $n$ & $\%$ & $n$ & $\%$ \\
\hline Nutrition content claims & 1966 & 79 & 2927 & 84 & 1180 & 85 & 6073 & 82 \\
\hline All health claims & 524 & 21 & 567 & 16 & 203 & 15 & 1294 & 18 \\
\hline High-level health claims $\dagger$ & 1 & 0.04 & 24 & 0.7 & 2 & 0.1 & 27 & 0.4 \\
\hline Total claims & 2490 & & 3494 & & 1383 & & 7367 & \\
\hline
\end{tabular}

*May not add to 100 due to rounding.

tThis is a subset of all health claims.

\section{Summary of results from 2011 study}

Across three categories, 1028 products were analysed: 598 beverages, 264 breakfast cereals and 166 cereal bars. Overall, $66 \%$ ( $n$ 679) products carried at least one claim ${ }^{(7)}$.

A higher proportion of products carried nutrition content claims than health claims: breakfast cereals ( $83 v .38 \%)$, cereal bars (77v. 14\%) and beverages (54v. 10\%) (Table 1).

Of products carrying health claims, $31 \%$ did not meet the NPSC. Of products carrying nutrition content claims, $29 \%$ did not meet the NPSC although that is not a requirement of Standard 1.2.7 (Table 1).

\section{Comparative analysis 2011-2016}

There was a large increase in the total number of products carrying claims in 2016 (see Table 1). Compared with 2011, there were also significantly higher proportions of products carrying claims, both overall $(P<0 \cdot 001)$ and in all three categories $(P=0.01, P<0.001$ and $P<0.001$ for beverages, cereals and cereal bars, respectively). There were significantly higher proportions of products carrying nutrition content claims in all three categories $(P=0.04, P<0.001$ and $P<0.001$ for beverages, cereals and cereal bars, respectively), and health claims in beverages $(P=0.02)$ and cereal bars $(P<0.001)$.

The proportions of products carrying claims that did not meet the NPSC did not change in beverages or breakfast cereals. However, there was a significant reduction in the proportion of cereal bars carrying claims that did not meet the NPSC for any claim $(P=0.01)$ and nutrition content claims $(P=0 \cdot 01)$. Despite this, there was a large increase in the number of products that carried claims and did not meet the NPSC.

There were no changes in the proportion of products carrying high-level health claims, both in total and those not meeting the NPSC. Contrary to the other claim types, there was little change in the total number of products carrying high-level health claims.

\section{Discussion}

Our study found that from 2011 to 2016, there was a significant increase in the number and the proportion of breakfast cereal, cereal bar and beverage products carrying nutrition content and health claims. The number of products carrying health claims that did not meet the NPSC more than doubled from 2011 to 2016, though the increase in proportion of products was non-significant (34v. $31 \%$ in 2011). These products are potentially in breach of Standard 1.2.7 as products carrying health claims are required to meet the NPSC ${ }^{(3)}$. Standard 1.2.7 is monitored by authorities in Australian states and territories, and enforcement actions can range from warning letters to prosecutions ${ }^{(14)}$. Data on enforcement actions are not publicly available.

We had anticipated that the introduction of the standard may have seen a decrease in the proportion of products carrying health claims due to the more stringent requirements of Standard 1.2.7; however, this has not been the case. A study of claims on chips and sweet biscuits in sixteen countries found that countries with health and nutrition content claim regulation had more products with claims than countries without regulation; the authors

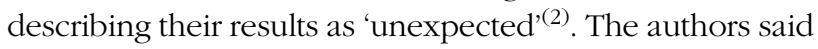
this could be due to higher literacy and health literacy in those countries leading to food companies responding with more claims on labels ${ }^{(2)}$. Other explanations could be that once regulation is in place, it is seen as approval for the use of claims on products or it could be because countries that had high numbers of claims saw a greater need to regulate. A random sample of a wider range of foods in five European countries found, although foods that carried claims were on average healthier, $30 \%$ of those carrying health claims did not pass the NPSC, noting this is not a requirement within European Union regulation ${ }^{(15)}$. In Canada, comparison of the prevalence of nutrition claims on products in a large and comprehensive database found a decrease from 2010 to 2013, although there had been changes in regulations and guidance documents in that time ${ }^{(16)}$. The study found, like our study, nutrient content claims were the most common type of claim on food products. Another study, using the NPSC and the same 2013 database, found $22 \%$ of products carrying health claims did not pass the NPSC, noting that Canadian regulation does not have a nutrient profiling requirement ${ }^{(17)}$.

It was beyond the scope of this project to determine whether the claims being made on labels in this study meet 
the other requirements of Standard 1.2.7, such as having sufficient evidence to confirm each food-health relationship or having sufficient levels of the nutrients being claimed $^{(3)}$. This is an area for future research.

It appears that since the introduction of Standard 1.2.7, there has been a proliferation of nutrition content claims, with significantly higher proportions of products within each category carrying nutrition content claims. Recent research has shown that consumers do not interpret nutrition content claims in the same way that food regulators $\mathrm{do}^{(18)}$. Specifically, if consumers were previously aware of nutrient-health relationships (e.g. the link between Ca and strong bones), they may interpret nutrition content and health claims in the same manner and infer health benefits of nutrition content claims ${ }^{(18,19)}$. This is potentially misleading, as consumers may choose to purchase products based on these inferences, regardless of the healthiness of the products ${ }^{(19,20)}$. Under Standard 1.2.7, nutrition content claims are not required to meet the NPSC; however, to ensure only healthier foods are eligible to carry claims, we are calling for regulation of nutrition content claims to include a requirement to meet the NPSC. This is especially important considering the increased number and proportions of products with nutrition content claims found in this study.

Nutrition content claims most commonly reflected added ingredients such as vitamins, wholegrains and fibre in the case of cereals and cereal bars and low in sugar claims in the case of drinks. Within health claims, there were common themes across the categories of energy and vitality and general health and well-being reflecting 'feel good' marketing and trends towards more healthconscious consumerism ${ }^{(20,21)}$. Common health claims themes for beverages were hydration and detox/cleanse/ balance, and cereals had a range of different health claims including digestive health while cereal bars were the category that featured most of the emotional well-being claims.

Regulation of such claims is important as claims can provide a 'health halo' to products and influence consumers' perceptions of that product ${ }^{(20)}$. Consumers do not clearly distinguish between nutrition content and health claims ${ }^{(20)}$; therefore, regulation serves to protect consumers from marketing claims that are unsubstantiated and overstate the benefits of the food.

There are several limitations of our study. Due to resourcing, we only included three food categories previously identified as carrying claims and this limits generalisability to the entire food supply. However, this study replicates the methods and food categories from our previous study, providing useful comparisons. To gain a deeper understanding of the prevalence of claims and healthiness of products carrying them across the entire food supply, this study could be replicated in all food categories. For the NPSC calculation, some estimations of fibre were made. Every effort was made to limit error by comparing to similar products. Each NPSC was calculated considering various fibre levels to ascertain that in most cases it did not make a difference to the 'healthy/unhealthy' categorisation.

The food supply may have changed substantially in the years since the data collection was conducted in 2016; therefore, these results cannot be extrapolated to the present time. However, it is unlikely the present food supply differs regarding the frequency of claims given there have been no changes in regulation of the food supply. Due to the observational nature of the study, we cannot conclude that the changes in the prevalence or types of claims or whether products carrying claims met the NPSC were a result of the implementation of Standard 1.2.7. Despite this, monitoring any changes in the marketplace after the introduction of new regulation is important to identify potentially detrimental effects.

Finally, this research captures single time points, meaning it does not capture seasonal variations in products, or more recently introduced foods. It is unclear whether the increases in claims were on products that have been introduced since the first study, or on existing products, or both. As shown by the results, significant numbers of new products were available in 2016. Further analysis could identify whether new or existing products were more likely to carry claims and not meet the NPSC.

\section{Conclusion}

To our knowledge, our study is the first investigating changes in the prevalence of claims and healthiness of products carrying claims since the implementation of Standard 1.2.7 in Australia. Twenty-eight percentage of products with health claims did not meet the NPSC, meaning they could be in breach of Standard 1.2.7. The high prevalence of products carrying nutrition content claims, proportion of products carrying claims that do not meet the NPSC and consumers' tendency to infer health benefits from nutrition content claims warrant the regulation of all claims using the NPSC. This will ensure consumers are not misled by claims on unhealthy food products.

\section{Acknowledgements}

Acknowledgements: The authors would like to thank Jane Dibbs and Amy Pagotto for their assistance with quality assurance of the data. Financial support: This research received no specific grant from any funding agency, commercial or not-for-profit sectors. Conflict of interest: L.W.-C., W.L.W. and C.H. - none. Authorship: L.W.-C. and C.H. formulated the research question, designed the study and oversaw the project. R.L., C.T. and W.L.W. had input into study methods. R.L. and C.T. collected, entered 
and cleaned the data and conducted the preliminary analysis. All authors had input into the final analysis approach. L.W.-C. and R.L. wrote the draft manuscript. All authors reviewed and approved the final manuscript. Ethics of buman subject participation: Ethical approval was not required as the research was observational with no participants.

\section{References}

1. Williams P, Yeatman H, Ridges L et al. (2006) Nutrition function, health and related claims on packaged Australian food products-prevalence and compliance with regulations. Asia Pac J Clin Nutr 15, 10-20.

2. Mayhew AJ, Lock K, Kelishadi R et al. (2016) Nutrition labelling, marketing techniques, nutrition claims and health claims on chip and biscuit packages from sixteen countries. Public Health Nutr 19, 998-1007.

3. Australian Government (2017) Australia New Zealand Food Standards Code - Standard 1.2.7 - Nutrition, Health and Related Claims. Australia New Zealand Food Standards Code [Internet]. https://www.legislation.gov.au/Series/ F2015L00394 (accessed December 2018).

4. Wellard-Cole L, Watson WL, Hughes C et al. (2019) How effective is food industry self-substantiation of food-health relationships underpinning health claims on food labels in Australia? Public Health Nutr 22, 1686-1695.

5. Food Standards Australia and New Zealand (2018) Nutrient Profiling Scoring Calculator for Standard 1.2.7 Canberra: Food Standards Australia New Zealand. http://www.food standards.gov.au/industry/labelling/pages/nutrientprofiling calculator/Default.aspx (accessed December 2018).

6. Food Standards Australia New Zealand (2016) Nutrition, Health and Related Claims: Food Standards Australia New Zealand. http://www.foodstandards.gov.au/industry/labelling/ Pages/Nutrition-health-and-related-claims.aspx (accessed August 2019).

7. Hughes C, Wellard L, Lin J et al. (2013) Regulating health claims on food labels using nutrient profiling: what will the proposed standard mean in the Australian supermarket? Public Health Nutr 16, 2154-2161.

8. Pulker CE, Scott JA \& Pollard CM (2018) Ultra-processed family foods in Australia: nutrition claims, health claims and marketing techniques. Public Health Nutr 21, 38-48.
9. Al-Ani HH, Devi A, Eyles H et al. (2016) Nutrition and health claims on healthy and less-healthy packaged food products in New Zealand. Br J Nutr 116, 1087-1094.

10. Youl T (2018) IBISWorld Industry Report G4111 Supermarkets and Grocery Stores in Australia. Melbourne: IBISWorld.

11. Australian Government (2018) Australia New Zealand Food Standards Code - Standard 1.2.8 - Nutrition Information Requirements: Federal Register of Legislation. https://www.legislation.gov.au/Details/F2018C00944 (accessed August 2019).

12. Australian Government (2018) Australia New Zealand Food Standards Code - Standard 1.2.10 - Information Requirements - Characterising Ingredients and Components of Food: Federal Register of Legislation. https://www.legislation.gov.au/Details/F2018C00945 (accessed August 2019).

13. Australian Government (2015) Australia New Zealand Food Standards Code - Standard 1.2.4 - Information Requirements - Statement of Ingredients: Federal Register of Legislation. https://www.legislation.gov.au/Details/ F2015L00392 (accessed August 2019).

14. NSW Food Authority (2018) Audits and Compliance: NSW Government. http://www.foodauthority.nsw.gov.au/ip/ audits-and-compliance/compliance (accessed August 2019).

15. Kaur A, Scarborough P, Hieke S et al. (2016) The nutritional quality of foods carrying health-related claims in Germany, The Netherlands, Spain, Slovenia and the United Kingdom. Eur J Clin Nutr $\mathbf{7 0} 1388-95$.

16. Franco-Arellano B, Bernstein JT, Norsen S et al. (2017) Assessing nutrition and other claims on food labels: a repeated cross-sectional analysis of the Canadian food supply. BMC Nutr $\mathbf{3}, 74$.

17. Franco-Arellano B, Labonte ME, Bernstein JT et al. (2018) Examining the nutritional quality of Canadian packaged foods and beverages with and without nutrition claims. Nutrients 10, 832.

18. Hodgkins CE, Egan B, Peacock M et al. (2019) Understanding how consumers categorise health related claims on foods: a consumer-derived typology of healthrelated claims. Nutrients $\mathbf{1 1}, 539$.

19. van Trijp HCM \& van der Lans IA (2007) Consumer perceptions of nutrition and health claims. Appetite 48, 305-324.

20. Williams PG (2005) Consumer understanding and use of health claims for foods. Nutr Rev 63, 256-264.

21. Nielsen (2015) We Are What We Eat, Healthy Eating Trends around the World. USA: The Nielsen Company. 